\title{
Prevalência De Uso De Antidepressivos E Benzodoazepínicos Em Uma Estratégia De Saúde E Da Família Em Santa Cruz Do Sul - RS
}

\author{
Vieira, A.R.; Borges, D.T.; Pouey,, R.C.;
}

Apresentador: Alexandra Rech Vieira

\section{Resumo}

Introdução: Atualmente, pode ser observado

um aumento no número de pessoas que utilizam

antidepressivos e benzodoazepínicos. Apesar dessas

classes medicamentosas trazerem benefícios aos

pacientes quando utilizadas corretamente, quando utilizadas de forma indevida podem acarretar em graves consequências. Método: O presente estudo foi realizado pela análise retrospectiva dos prontuários dos pacientes da Estratégia de Saúde e da Família (ESF) Pedreira da cidade de Santa Cruz do Sul - RS, buscando a prevalência do uso de antidepressivos e benzodoazepínicos. Resultados: Dos 3599 prontuários analisados, foi encontrado uma prevalência de $5,5 \%$ de uso dessas classes farmacológicas, sendo a Flouxetina o mais utilizado. O medicamento com a menor incidência foi o Alprazolam. O perfil encontrado de pacientes usuários desse medicamento foi pacientes do sexo feminino, na faixa etária de 50-60 anos. Conclusão: Os dados encontrados durante esse estudo são equivalentes com os dados já relatados em outros estudos encontrados na literatura. É de suma importância que esses pacientes recebam um acompanhamento médico adequado tendo por finalidade evitar seus efeitos colaterais e possam ter uma melhor qualidade de vida.

\section{Referência:}

Vieira, A.R.; Borges, D.T.; Pouey,, R.C.;. Prevalência De Uso De Antidepressivos E Benzodoazepínicos Em Uma Estratégia De Saúde E Da Família Em Santa Cruz Do Sul - RS. In: Il Congresso Brasileiro de Medicina Hospitalar - II CBMH [= Blucher Medical Proceedings, vol.1, num.5] São Paulo: Editora Blucher, 2014. p.40

DOI 10.5151/medpro-II-cbmh-033 